\title{
Conservative Treatment Using Marsupialization for Cysts Occurring in the Jaw of Adolescents: A Case Report
}

\author{
Sun-Ung Yun, Hai-Won Jung, Byung-yong Cho, Byung-Joon Choi, \\ Baek-Soo Lee, Yong-Dae Kwon, Joo-Young Ohe, Jung-Woo Lee \\ Department of Oral and Maxillofacial Surgery, Kyung Hee University School of Dentistry, Seoul, Korea
}

\begin{abstract}
Dentigerous cyst (DC) is an odontogenic cyst associated with the crown of an impacted, embedded, unerupted, or developing tooth. It is the second most common type of odontogenic cysts, accounting for $14 \%$ to $24 \%$ of all jaw cysts. Although these cysts occur more frequently during the second and third decades of life, they can also be found in children and adolescents in the mixed dentition stage. Treatment of the odontogenic cyst involves enucleation or marsupialization/decompression methods. The latter approach is preferred for larger cysts, and it is especially helpful in adolescent patients in conserving the unerupted permanent successors. The aim of treatment for DC is the complete elimination of pathology and maintenance of dentition with minimal surgical intervention. Recently defined criteria for the selection of treatment modality include the cyst size and location of the cyst, patient age, dentition involved, stage of root development, position of the tooth involved in the jaw, and relationship with the surrounding vital structure. Marsupialization or decompression technique has been advocated by several authors for treating DCs among young patients. In this conservative technique, the creation of an accessory cavity helps relieve intra-cystic pressure and accelerate the healing of cystic lesion. This technique has been successfully performed and is indicated for growing children and adolescents. Here, we report a large cyst lesion in the mandible treated by marsupialization for conservative management. In conclusion, successful reduction in size was achieved, and intraoperative complication could be prevented.
\end{abstract}

Key Words: Decompression; Dentigerous cyst

Corresponding Author: Byung-Joon Choi

Department of Oral and Maxillofacial Surgery, Kyung Hee University Dental Hospital, 26 Kyungheedae-ro, Dongdaemun-gu, Seoul 130-701, Korea

TEL : +82-2-958-9440, FAX : +82-2-966-4572, E-mail : sjnb@khu.ac,kr

Received for publication April 10, 2014; Returned after revision June 9, 2014; Accepted for publication June 16, 2014

Copyright $\odot 2014$ by Korean Academy of Dental Science

(c) This is an open access article distributed under the terms of the Creative Commons Attribution Non-Commercial License (http://creativecommons.org/licenses/ by-nc/3.0) which permits unrestricted non-commercial use, distribution, and reproduction in any medium, provided the original work is properly cited. 


\section{Introduction}

As one of the most common odontogenic cysts occurring in the jaw, dentigerous cyst (DC) is formed due to the separation of crown and dental follicle caused by the inflow of liquid into the dental follicle surrounding the anatomical crown of unerupted tooth or developing tooth ${ }^{1)}$. The cause of disease has not been clearly identified, but it is known to occur due to the accumulation of exudation in the space between the crown of the unerupted tooth and reduced enamel epithelium. That is why there is an unerupted tooth inside of the cyst in many cases. The wall of the cyst is usually attached to the cement-enamel junction of the tooth. It can grow big enough to invade into the whole jaw, sometimes causing the swelling of cortical bone ${ }^{2)}$.

Clinically, it is usually painless. If it becomes infected; however, it can cause pain and tumentia. The more affected age group is young people in their teens or 20s. There is little gender differentiation, but it occurs a bit more frequently in men. It develops anywhere in the jaw but occurs frequently in the mandible; mandibular third molar, maxillary canine, maxillary third molar, and mandibular second premolar are the more affected areas ${ }^{3)}$.

Radiologically, it is a unicystic radiolucent lesion including the crown of unerupted tooth; its boundary is clear, with sclerotic border with bone shown sometimes. The cyst can grow, invade into the nasal cavity, maxillary sinus, orbital floor, condyle, and mandibular ramus, and ause the displacement of neighboring teeth or root resorption. The wall of the cyst is lined with thin non-keratinized epithelium and composed of loose fibrous connective tissue including glycosaminoglycan. If infected, it causes the thickening of the epithelial lining and the overgrowth of rete ridge $^{4)}$.

As treatments, enucleation and marsupialization are recommended; the treatment method is decided depending on the size of the cyst, correlation between the cyst and surrounding tissues, and preservation of related teeth. Marsupialization is a conservative technique that opens part of the cyst wall to intrabuccal and reduces the size of lesion; it can be used in the treatment of cysts in the jaw such as DC, ameloblastoma, and odontogenic keratocyst or cystic odontogenic tumor ${ }^{5,6)}$.

As a result of the clinical and radiological tests of adolescent patients who visited the Department of Oral Surgery at Kyung Hee University Dental Hospital (Seoul, Korea), we judged as DC those occurring in the maxilla or mandible and reduced the cyst size by performing marsupialization first followed by enucleation to minimize bone defect. Since they showed successful treatment result, we report such cases.
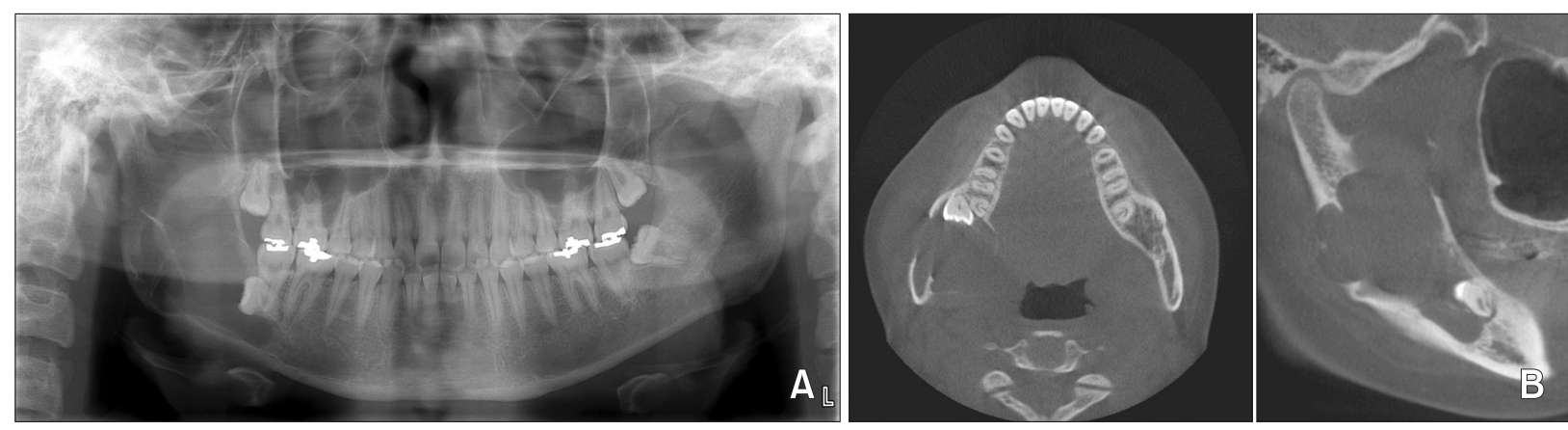

Fig. 1. The initial panoramic view (A) and computed tomographic scan (B) shows well defined multilocular radiolucent lesion on \#48 to right mandibular ramus, expansion of mandibular inferior border and displacement of \#48. 


\section{Case Report}

\section{Case 1}

A female patient aged 17 years visited our hospital due to tumentia in the mandibular right second and third molars. There was no significant medical history. In palpation, induration was detected, with the mandibular right second molar showing no percussion response or mobility. Based on the radioactive examination, an extensive radiolucent lesion accompanying the swelling of the mandible was observed from the mandibular right third molar to the mandibular ramus, with the mandibular right third molar impacted inside of the lesion (Fig. 1). We performed marsupialization in the centrifugal area of the mandibular right second mola and subsequently maintained the tunnel by inserting silastic penrose drain. In addition we conducted regular examination at one-week intervals for the first three months, and then at onemonth intervals for eight months (Fig 2). Ten months later, the patient complained of discomfort in the area of the mandibular right third molar, which erupted to the occlusal surface. Therefore, we performed surgical extraction and biopsy again (Fig. 3). Two and a half years later, we confirmed that the size of the cyst had been reduced and performed cyst enucleation under topical anesthesia. The final result of the biopsy confirmed that it was a DC (Fig. 4).

\section{Case 2}

A male patient aged 13 transferred to our hospital

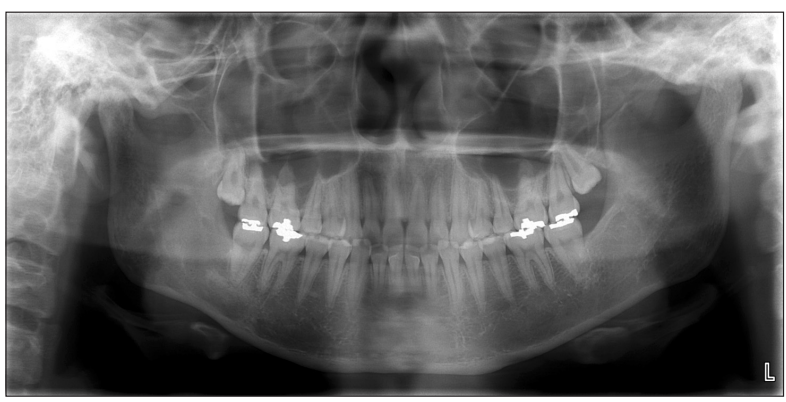

Fig. 3. The panoramic view after 10 months shows that the mandibular right third molar was extracted.
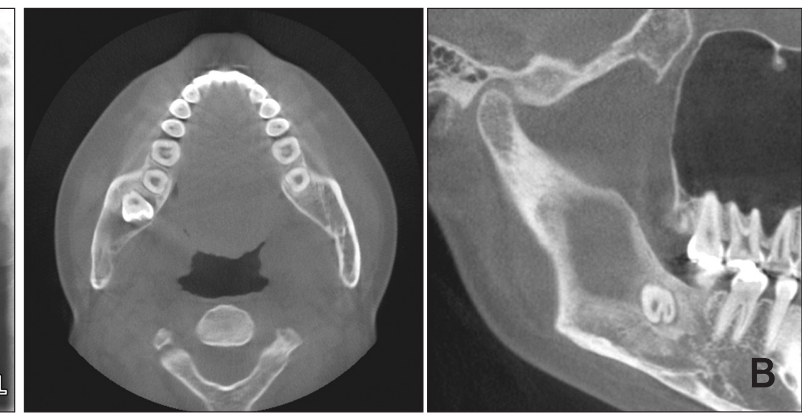

Fig. 2. (A) The panoramic view after 6 months shows that the size of the cyst on right mandibular area had been reduced. (B) Computed tomographic scan after 6 months.
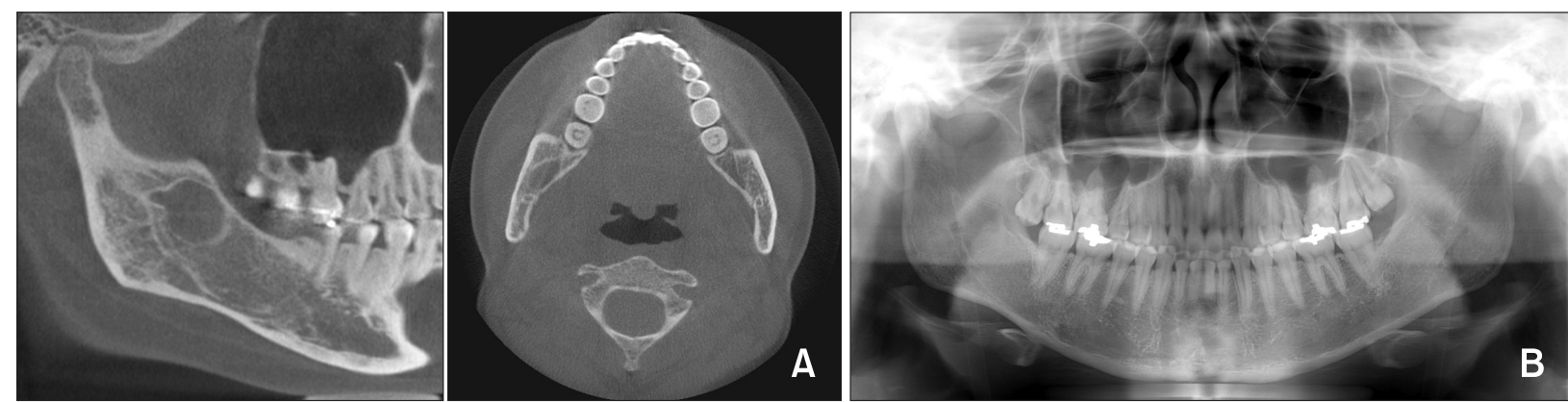

Fig. 4. (A) Computed tomographic scan after 30 months. (B) One year after operation. 

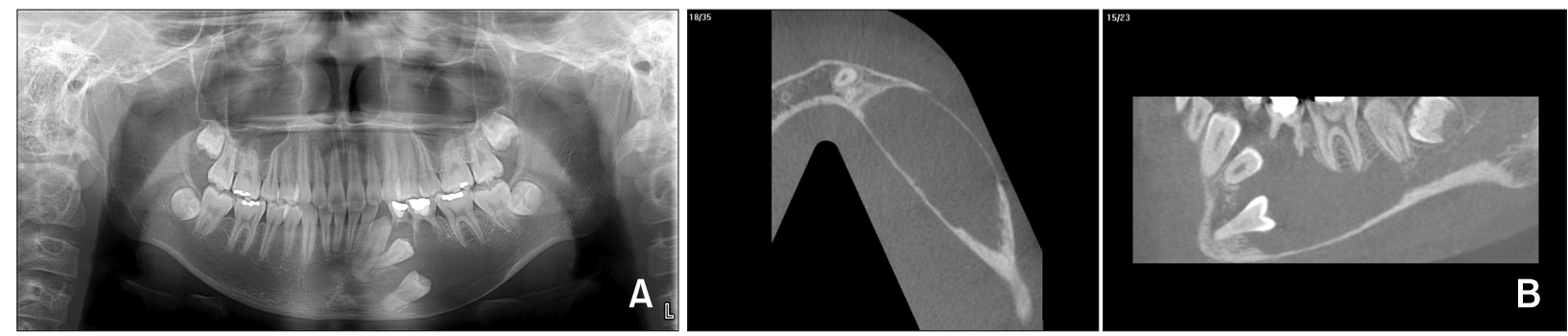

Fig. 5. The initial panoramic view (A) and computed tomographic scan (B) shows the poorly defined radiolucent lesion on \#74 \#37 area with thinning and expansion of inferior mandibular border and displacement on \#34, \#35 and external root resorption on \#74, \#75.

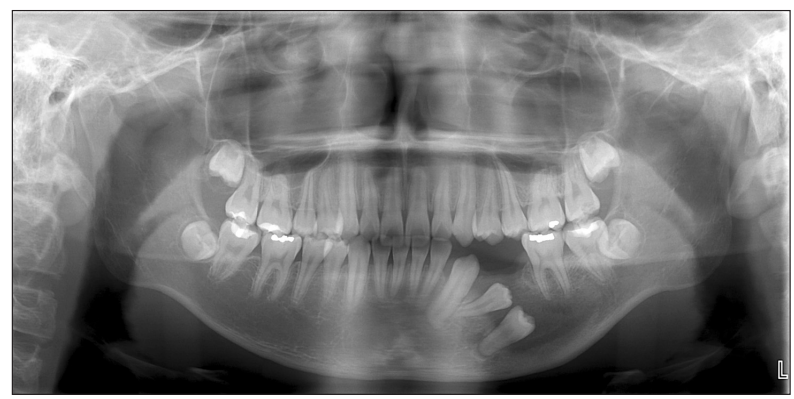

Fig. 6. The panoramic view after 6 months shows that the size of the cyst on left mandibular area had been reduced.

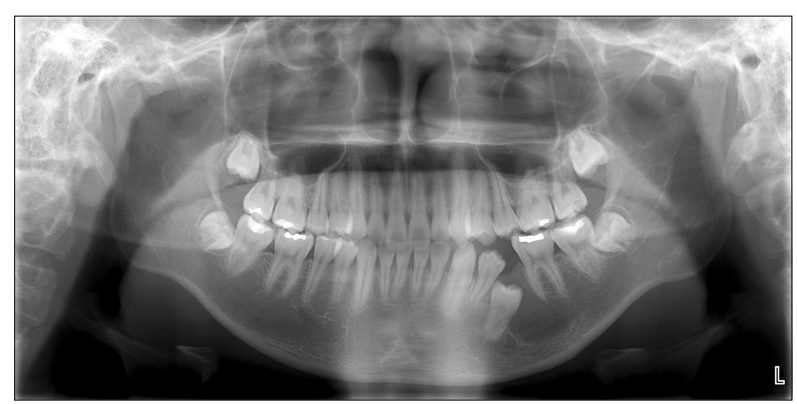

Fig. 7. The panoramic view after 24 months shows that the size of the cyst on left mandibular area had been reduced.

from a dental clinic due to a cyst in the mandibular anterior region. There was no significant medical history. Tumentia was observed in the buccal area of the left mandible, and there was also an induration. The mandibular left first molar showed percussion response, whereas the mandibular left first and second primary molars exhibited mobility. There was a fistula formed in the area of the mandibular left canine, and it was being drained. Based on the radioactive examination, a radio-

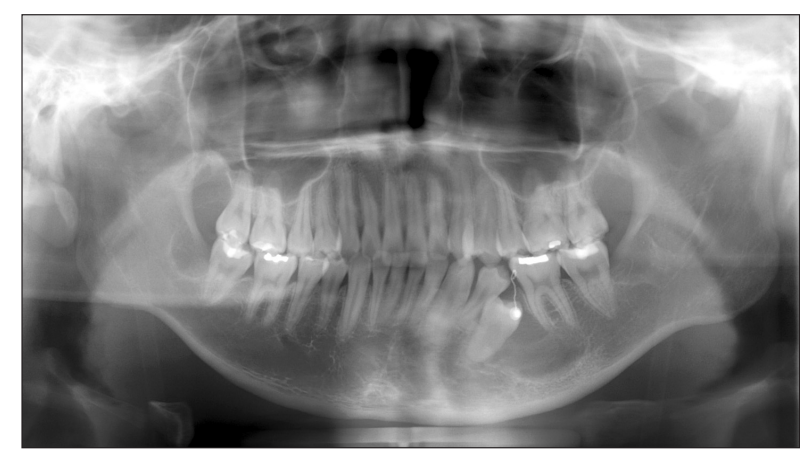

Fig. 8. The panoramic view after 48 months shows that the size of the cyst on left mandibular area had been reduced.

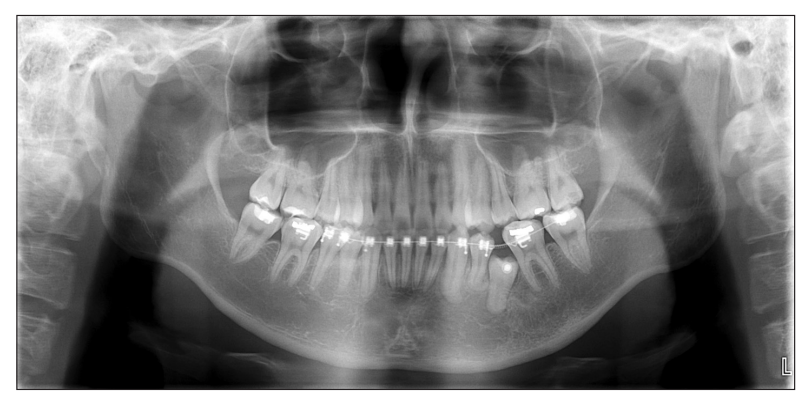

Fig. 9. The mandibular left second premolar was completely erupted through orthodontic traction on pasting 17 months after operation.

lucent lesion accompanying the swelling of the mandibular inferior border from the mandibular left first primary molar to the mandibular left first molar; the mandibular left first and second premolars were unerupted (Fig. 5). We extracted the mandibular left first and second primary molars, and then opened from the extraction socket to the cyst. After that, we performed marsupialization using silastic penrose drain and conducted regular 


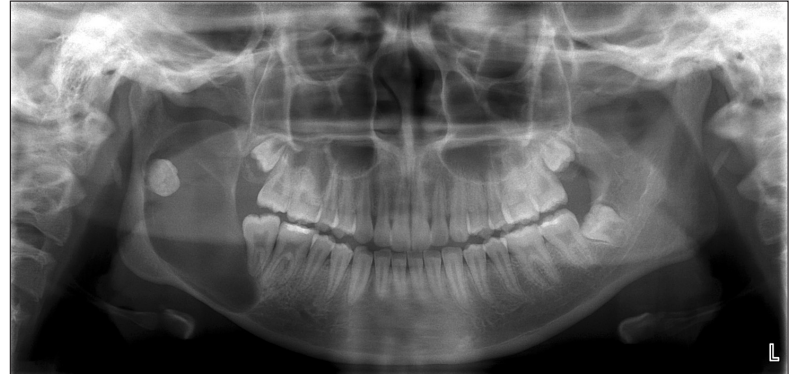

Fig. 10. The initial panoramic view shows well defined monolocular radiolucent lesion from \#37 to sigmoid notch area with corticated margin and smooth border.

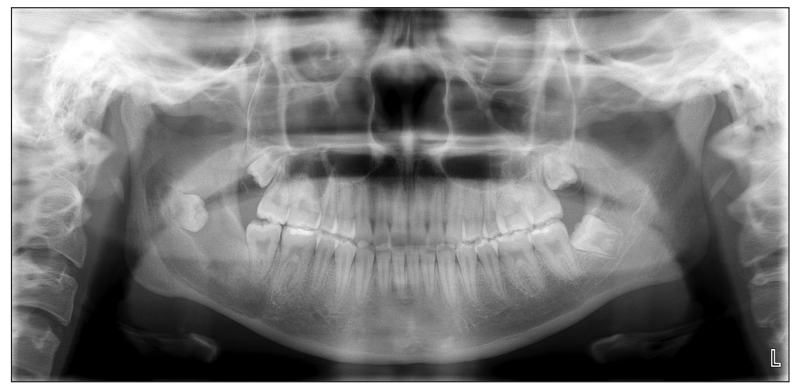

Fig. 11. The panoramic view after 12 months shows that the size of the cyst on right mandibular area had been reduced.

examination at one-week intervals for the first three months, and then at two-week intervals for two years (Fig. 6). Two years later, it was observed that the impacted mandibular left first and second premolars were naturally erupted (Fig. 7). After regular examination for three and a half years, we performed cyst enucleation and crown exposure of the mandibular left second premolar under general anesthesia. The final result of the biopsy confirmed that it was a DC (Fig. 8). After that, we induced the complete eruption of the mandibular left second premolar through orthodontic traction (Fig. 9).

\section{Case 3}

A female patient aged 14 visited our hospital due to pain in the areas of the temporomandibular joint and mandibular ramus. There was no significant medical history. We could see gingival edema from the mandibular right first molar to third molar and swelling in the buccal area of the right side. There

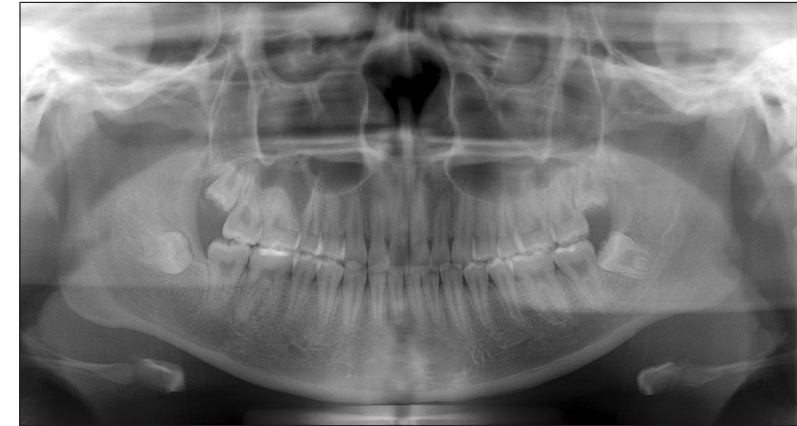

Fig. 12. The panoramic view after 24 months shows that the size of the cyst on right mandibular area had been reduced and the impacted right mandibular third molar moved to the occlusal surface.

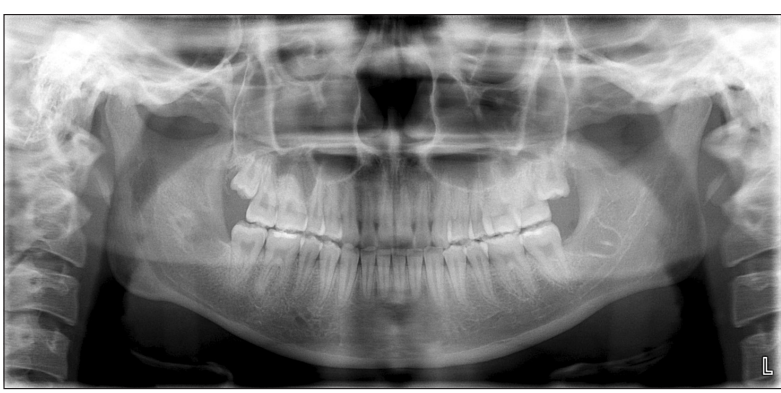

Fig. 13. Post-operation panoramic view.

was mouth opening limitation accompanying pain as well. Based on the radioactive examination, a radiolucent lesion was detected from the mandibular right first molar to the sigmoid notch, with the mandibular right third molar impacted inside of the lesion (Fig. 10). After bone removal in the centrifugal area of the mandibular right second molar, we performed marsupialization and subsequently maintained the tunnel by insertiing silastic penrose drain. In addition, we conducted regular examination at one-week intervals for the first three months, and then at two-month intervals for one year (Fig. 11). During the two-year observation, the size of the cyst was reduced, and the impacted mandibular right third molar moved from the area of the mandibular ramus to the location of the occlusal surface (Fig. 12). After that, we performed cyst enucleation under general anesthesia and extracted the mandibular right third molar (Fig. 13). 


\section{Discussion}

DC occurs in relation to unerupted tooth or impacted tooth inside bone. Main ${ }^{7)}$ and Benn and Altini $^{8)}$ divided the DCs into two types based on the pathogenesis. One type is the developmental DC caused by a mature impacted tooth ${ }^{9)}$. This is usually detected by chance in patients in their late teens or early 20s, and most of them are found in the mandibular third molarv ${ }^{10)}$. Another type is inflammatory, originating with immature tooth. The cause is usually the infection of devitalized milk tooth bud. For this reason, this type of cyst occurs in patients in mixed dentition, and most of the patients visit the hospital due to pain and tumentia in the area involved ${ }^{11)}$. Since it occurs without particular clinical symptom, if a DC is found late, the cyst can increase considerably and invade into the nasal cavity, maxillary sinus, orbital floor, coracoid, condyle, and mandibular ramus. If the mandibular molar is included in the cyst, it can cause the displacement of the mandibular inferior border or ascending ramus, expansion of cortical bone through invasion into neighboring tissue, and, even worse, malocclusion and facial asymmetry through tooth displacement $t^{3)}$. Moreover, the second infection of the cyst can destroy neighboring structure and cause a serious problem through the variation to malignant tumor from the cyst wall ${ }^{33}$.

In these cases, unicystic radiolucent lesion related to unerupted permanent tooth or impacted mandibular third molar was found, and its boundary was clear. In case 2, displacement of the mandibular left canine and the mandibular left first mola was observed; in case 3, invasion into coracoids was detected. It could be diagnosed as ameloblastoma, calcifying odontogenic cyst, adenomatoid odontogenic tumor, or ameloblastic fibroma. In these cases, however, they are confirmed to be the DC through biopsy before performing marsupialization ${ }^{12)}$.

The treatment purpose of the cyst is to preserve healthy teeth by removing the abnormal lining in the jaw and making unerupted tooth function normally. In addition, it should preserve neighboring major structures such as maxillary sinus and inferior alveolar nerve and maintain the original shape and function of the area involved. ${ }^{11}$ Clinical techniques include cyst enucleation, marsupialization, which turns cyst lumen into oral part, and mixture of cyst enucleation and marsupialization wherein the cyst size is reduced by marsupialization and the cyst is subsequently enucleated $^{13)}$.

The treatment method of DC is decided depending on the size and location of the cyst, correlation between the cyst and surrounding structures, and vitality of permanent tooth buds. If no complications are expected after surgery, enucleation can be performed $^{14)}$. As a result of prognosis observation after the marsupialization of cysts accompanying serious displacement of tooth or extensive cysts, however, change into tumor from the cyst wall has rarely been reported. In 1970, Stanback ${ }^{15)}$ said that marsupialization was valuable as a primary technique for children, enhancing the preservability of permanent teeth and neighboring structures.

The merits of marsupialization include simple surgery, no damage to neighboring tissues, no risk of palatal fistula, and prevention of unnecessary loss of permanent teeth in case of large DC of child and adolescent patients in particular ${ }^{11)}$. When treated with marsupialization, however, the patient has to visit the hospital regularly to be observed since the possibility of developing into a tumor cannot be discounted ${ }^{6}$. In these cases, we performed marsupialization because damage to neighboring structures was a concern owing to the big size of the cyst. In addition, the teeth inside of the cyst had to be preserved since the patients were young. As a result of marsupialization, the cyst disappeared without damage to neighboring major structures. In case 2, we could also observe the natural eruption of the permanent teeth involved inside of the cyst and the return of the displaced neighboring teeth to their 
original position.

Therefore, when the developing teeth of adolescents are included in the cyst, preserving the teeth involved with the cyst using marsupialization is considered advisable. Also, even if the displacement of unerupted teeth in relation to the cyst is evident, observing first before the extraction or orthodontic traction is deemed desirable.

When the cyst is treated with marsupialization, the patient has to visit the hospital regularly for observation or receive additional enucleation since the cyst can recur or develop as tumor.

\section{Conflict of Interest}

No potential conflict of interest relevant to this article was reported.

\section{References}

1. Manor E, Kachko L, Puterman MB, Szabo G, Bodner L. Cystic lesions of the jaws - a clinicopathological study of 322 cases and review of the literature. Int J Med Sci. 2012; 9: 20-6.

2. Ziccardi VB, Eggleston TI, Schneider RE. Using fenestration technique to treat a large dentigerous cyst. J Am Dent Assoc. 1997; 128: 201-5.

3. Browne RM. The pathogenesis of odontogenic cysts: a review. J Oral Pathol. 1975; 4: 31-46.

4. Shafer WG, Hine MK, Levy BM. A textbook of oral pathology. 4th ed. Philadelphia: Saunders; 1983. p.260-5.
5. Waldren CW. Conservative two stage procedure to prevent deformity and loss of useful teeth. Am J Orthod. 1941; 27: 313.

6. Jacobi R. Spontaneous repositioning of displaced molars after marsupialization of a dentigerous cyst. J Am Dent Assoc. 1981; 102: 655-6.

7. Main DM. Epithelial jaw cysts: 10 years of the WHO classification. J Oral Pathol. 1985; 14: 1-7.

8. Benn A, Altini M. Dentigerous cysts of inflammatory origin. A clinicopathologic study. Oral Surg Oral Med Oral Pathol Oral Radiol Endod. 1996; 81: 203-9.

9. Sapp JP, Eversole LR, Wysocki GP. Contemporary oral and maxillofacial pathology. 1st ed. St. Louis: Mosby; 1997. p.39-43.

10. Lucas RB. Pathology of tumors of the oral tissues. 1st ed. London: Churchill Livingstone; 1964. p.260.

11. Laskin DM. Oral and maxillofacial surgery. St. Louis: Mosby; 1985. p.427-86.

12. Martensson G. Cysts and carcinoma of the jaws. Oral Surg Oral Med Oral Pathol. 1955; 8: 673-81.

13. Goaz PW, White SC. Oral radiology: principles and interpretation. 3rd ed. St. Louis: Mosby; 1994. p.400-4.

14. Killey HC, Kay LW, Seward GR. Benign cystic lesions of the jaws, their diagnosis and treatment. 3rd ed. Edinburgh; New York: Churchill Livingstone; 1977. p.18-61.

15. Stanback JS 3rd. The management of bilateral cysts of the mandible. Oral Surg Oral Med Oral Pathol. 1970; 30: 587-91. 